\title{
Data diversity
}

Understanding how humans behave, why we behave the way we do, what the consequences of our behaviour are and how behaviour can change are complex, multi-faceted questions, addressed by numerous diverse disciplines that rely on different methods and types of data. We welcome them all in the journal.

N ature Human Behaviour is celebrating its second birthday this month. As a new journal, we are frequently approached with the question, 'Will the journal consider my type of research?'. We take a very broad view of what the scope of the journal is and the short answer to this question is 'almost certainly yes'.

Our aim is to publish outstanding research on how humans behave and why we behave the way we do. We consider research that explains the behaviour of individuals, as well as of collectives. We also prioritize for publication research that identifies the consequences of human behaviour, as well as how behaviour can change for individual or collective benefit. There is a vast range of data types that are instrumental in addressing these questions across different fields that span the social, biological, health and physical sciences, and these data are not derived from a single approach or source. As a multidisciplinary journal, Nature Human Behaviour is not partial to any individual field's approach to measuring and understanding human behaviour.

The journal publishes both primary and secondary data; welcomes both quantitative and qualitative research, as well as purely theoretical contributions. Approaches are not limited to any single type of research, such as lab-based experiments, or conversely, analysis of aggregate or macrodata, but span the entire breadth of data sources (Fig. 1).

Behavioural data come in many different forms. From button presses, eye movements, narrative responses, diaries, interviews and surveys, all the way to ethnographic observations, actual health and financial decisions, voting decisions, consumer behaviour data and more, the wealth of data types matches the complexity and uniqueness of the subject matter. In some cases, the data are obtained in real time; in others, researchers look back at traces of behaviour left behind deep in our historical past - for instance, in the form of artefacts - or much more recently, for instance, in our online interactions, where data ranges from the things we re-tweet to the number of our friends on social media platforms. Each of these data types can illuminate the how and why of human behaviour from

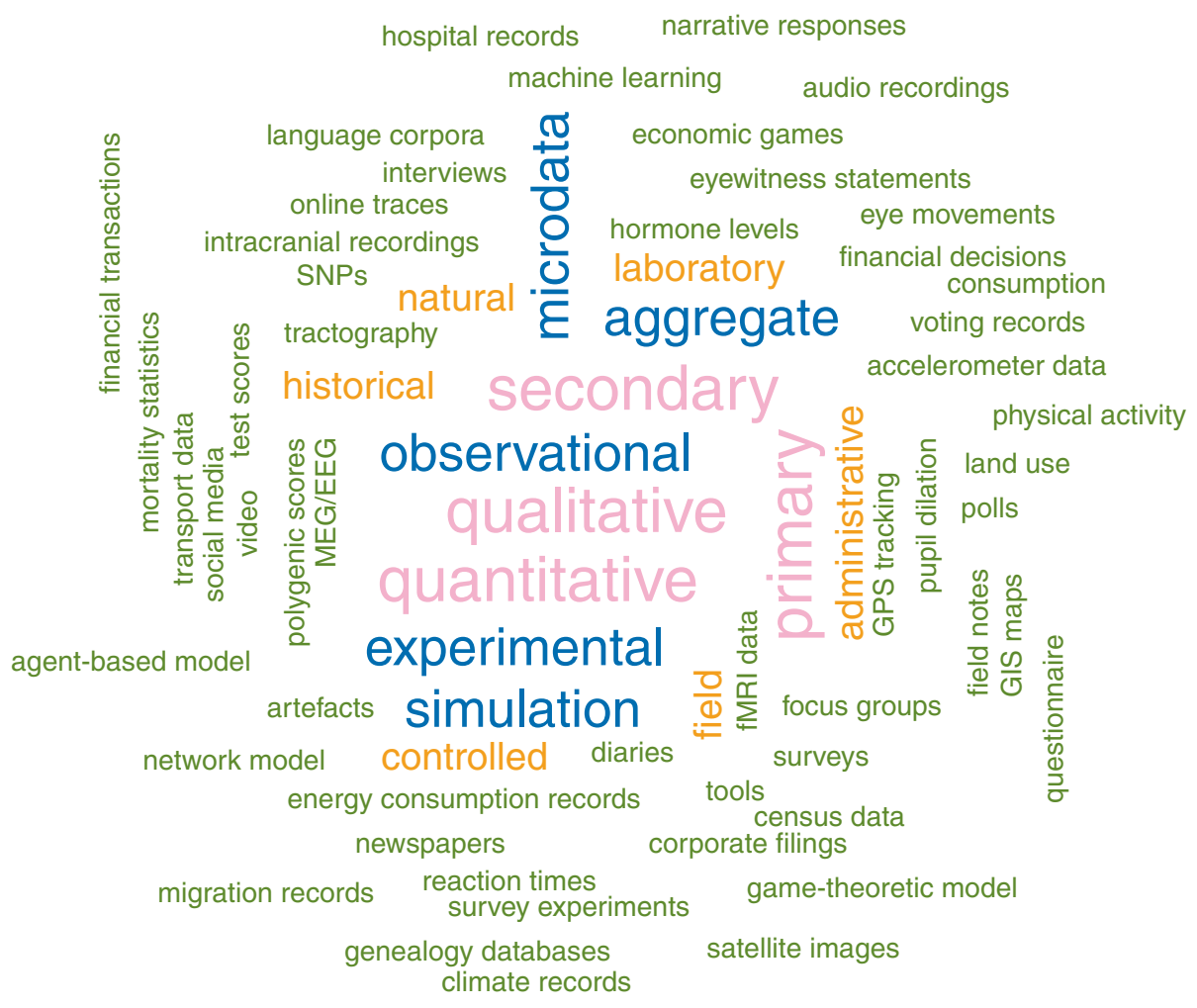

Fig. 1 | We welcome research that uses any type of data to address key questions about individual and collective human behaviour, its social and biological causes, as well as its consequences. Types of data are examples, rather than an exhaustive list.

different disciplinary perspectives and levels of analysis.

Understanding human behaviour requires going far beyond direct measurements of behaviour under experiment or observation. Understanding collective behaviour and dynamics, for instance, requires large-scale administrative data, data extracted from the historical record, and aggregate or macrodata. These types of data - such as census data, economic indicators, conflict records, migration records, crime statistics - allow researchers in different fields to answer fundamental questions regarding the determinants and consequences of human behaviour such as, 'What makes wars deadlier?', 'How can crime be reduced?' and 'What are the causes of income disparities among ethnic groups?'. In health research, measures including longitudinal cohort data, population health statistics, case studies, health surveillance data, and data assembled in biobanks provide invaluable sources of information to understand human welfare and mortality. In human geography and environmental science, answering key questions about the social forces shaping behaviour or predicting the consequences of behaviour involves access to large-scale environmental and geospatial data - GIS maps, satellite images, climate statistics, land- and resource-use data - combined with aggregate socioeconomic data.

Biological data, in contrast, provide a different level of explanation of human behaviour. Neural data, for example, can offer insights into the computations underlying human behaviour, including how we process and represent information 
and how that in turn affects our memories, decisions and actions. Genetic and genomic data can be used not only to understand the precursors for individual differences (for example, why are some people more at risk for post-traumatic stress disorder than others? Is there a genetic contribution to individual differences in educational attainment?), but also to reveal the biological consequences of behaviour (for instance, what are the consequences of assortative mating on the genome?). The scope of biological data used to build and validate accounts of human behaviour is extensive. To name but a few: skin conductance and pupil dilation are measures of uncertainty or fear in psychological studies; brain imaging is not purely focused on recordings of neural activity but also offers precise maps of differences and disease-related changes in brain morphology, used in psychiatric and neuropsychological research. Biological samples such as saliva, blood, bone and teeth taken off the living or the long-deceased help us to understand the conditions under which we thrive or perish.

Simulation data cut across fields, disciplines and sciences. The product of mathematical or computational models, simulation is used to formalize predictions, and verify accounts of how and why humans behave the way we do. Simulations are uniquely flexible, because they can be applied to a very wide range of data and validated in both synthetic and real-world data. This way, simulation has become a tool for theory building and testing that lies at the heart of confirmatory research into any aspect of human behaviour, ranging from the signals of cells to the actions of nations.

Nature Human Behaviour is defined by its theme, not by methodology or data type. There is a vast array of data types that speaks to the 'how' and 'why' of human behaviour at different levels of explanation, and we welcome submissions from all walks of science, regardless of method or data type. The richer and more diverse, the better.

Published online: 11 January 2019 https://doi.org/10.1038/s41562-018-0525-y 